\title{
Aerodynamic and impact thresholds for cohesive mixture of sand and non-volatile liquid
}

\author{
Jean-Baptiste Besnard ${ }^{1,2, *}$, Alexandre Valance ${ }^{1}$, Pascal Dupont ${ }^{2}$, and Ahmed Ould El Moctar ${ }^{3}$ \\ ${ }^{1}$ Univ Rennes, CNRS, Institut de Physique de Rennes, UMR 6251, 35042 Rennes, France \\ ${ }^{2}$ Univ Rennes, INSA Rennes, LGCGM, 35043 Rennes, France \\ ${ }^{3}$ Univ Nantes, CNRS, Laboratoire Thermique et Energie, UMR 6607, 44306 Nantes Cedex, France
}

\begin{abstract}
The moisture has been recognized to have a significant influence on the initiation movement of sand by wind and consequently on sand transport rates. The pertinent literature regarding these phenomena is sparse and current available theoretical and empirical models exhibit considerable disagreement regarding the magnitude of moisture effects. We believe that these discrepancies comes from the fact that the moisture levels are not well controlled neither properly measured and are susceptible to strongly vary over time due to evaporation. To get rid of the variability of moisture content due to evaporation, we propose a new approach based on the use of non-volatile liquid, namely silicon oil instead of water. This insures a proper control of the liquid content and the production of reliable data concerning the variation of the transport threshold with liquid content.
\end{abstract}

\section{Introduction}

Aerodynamic and impact thresholds are a key ingredient for aeolian sand transport description. The dependence of these thresholds with soil moisture is still poorly understood and there is a need for well-controlled experiments to improve our understanding.

For a dry sand soil, transport is initiated when the friction speed overpasses a critical value $u_{* t d}$ which depends on the grain diameter $D$ and particle density $\rho_{p}[1]$ :

$$
u_{* t d}=A\left[\frac{\left(\rho_{p}-\rho_{a}\right)}{\rho_{a}} g D\right]^{0.5}
$$

Where $A$ is an empirical coefficient [2]. Typical value of the aerodynamic threshold for dry sand is $u_{* t d}=$ $0.21 \mathrm{~m} \cdot \mathrm{s}^{-1}$ for sand grains with mean diameter $D=$ $0,2 \mathrm{~mm}$ [3]. There exists another threshold corresponding to the cessation transport, the so-called impact threshold $u_{* i m p}$. The latter is smaller than the aerodynamic threshold $\left(u_{* i m p} \simeq 0.85 . u_{* t d}[4]\right)$ and is at the origin of hysteresis effect.

Numerous studies have been devoted to the quantification of the effect of moisture content on the aerodynamic threshold but available empirical models exhibit considerable disagreement with the magnitude of the moisture effect. For example, Belly [5] who studied this effect through wind-tunnel experiments proposed the following empirical relation:

$$
u_{* t w}=u_{* t d}\left(1.8+0.6 \log _{10} w\right),
$$

where $w$ is the gravimetric water content, while Hotta [6] provided an other empirical relation model with a linear

*e-mail: jean-baptiste.besnard@univ-rennes1.fr

A video is available at https://doi.org/10.48448/vqdr-7935 increase of the threshold friction speed with the water content:

$$
u_{* t w}=u_{* t d}+7.5 w I_{w}
$$

where $I_{w}$ is a correction factor taken account for the evaporation ( $I_{w}=1$ if there is no evaporation).

Theoretical models were also developed. From a force balance between the grain weight $P_{g}$, the cohesive force $F_{c}$ and the wind shear stress, McKenna-Neuman and Nickling [7] derived the following equation:

$$
u_{* t w}=u_{* t d} \cdot\left[1+\alpha B_{o}^{-1}\right]^{0.5}
$$

where $B_{o}=P_{g} / F_{c}$ is the dimensionless Bond number and $\alpha$ is a constant geometrical parameter given by

$$
\alpha=\left[\frac{2 \sin 2 \beta \cos \beta}{\sin \beta}\right]
$$

$\beta$ is the particle friction angle $\left(\beta \simeq 30^{\circ}\right.$ for a loose packing). To take benefit of Eq. 4, the knowledge of the cohesive force $F_{c}$ and its dependence with the water content is required, which is a challenge as well. Based on physical model considering capillary cohesive force, Kawata and Tsuchiya [8] derived a relation between $F_{c}$ and the water content $w$ suggesting that $F_{c} \propto \gamma \sqrt{w}$ (where $\gamma$ is the surface tension). As suggested by Hotta, we believed that the great diversity in the experimental data regarding the effect of the moisture on the aerodynamic threshold comes from the fact of the moisture levels are not well controlled and vary in course of time due to evaporation. Recent experiments [9] confirmed that for low moisture levels the transport is intermittent and is controlled by the evaporation process. Here, to get rid of the complexity of the evaporation phenomenon, we choose to work with cohesive sand 


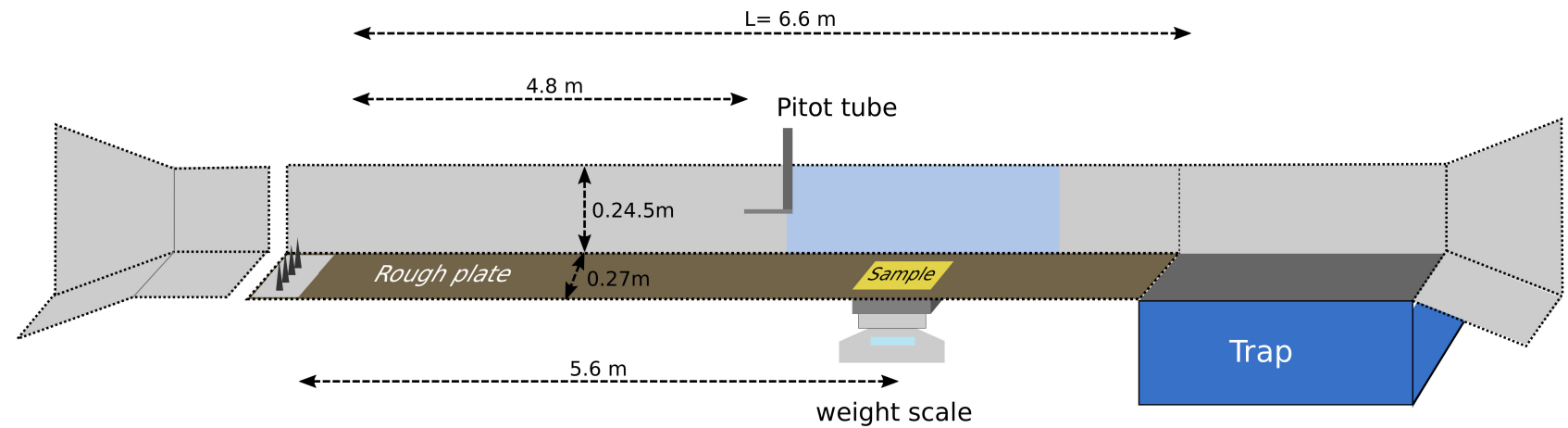

Figure 1. Schematic diagram of the wind tunnel facilities, with wind velocity measurement and weighting of the sample in time.

where the water is replaced by silicon oil, a non-volatile liquid for atmospheric conditions. This allows us to conduct well-controlled experiments in a wind-tunnel and to quantify how the aerodynamic threshold but also the impact threshold vary with the liquid content.

\section{Experimental setup}

We carried out experiments in a wind-tunnel which has a $6.6 \mathrm{~m}$ working length and a $0.245 \mathrm{~m} \times 0.27 \mathrm{~m}$ cross section (see Fig 1). We used as a cohesive sand bed a mixture of $200 \mu \mathrm{m}$ sand and silicone oil with a surface tension $\gamma=20.6 \mathrm{mN} \cdot \mathrm{m}^{-1}$. The portion of the wind-tunnel filled of this sand mixture is reduced to a square surface area of dimensions $0.15 \mathrm{~m} \times 0.15 \mathrm{~m}$ with a depth of $0.02 \mathrm{~m}$, either a mass of sand of $M=720 \mathrm{~g}$. The sand bed is contained in a box placed $5.4 \mathrm{~m}$ downstream the entrance of the wind-tunnel and its surface is at the same level as the wind-tunnel floor so that there is no discontinuity. The sand bed is weighed continuously during the experiment thanks to a high accuracy scale with $\Delta M_{\min }=1 \cdot 10^{-1} \mathrm{~g}$.

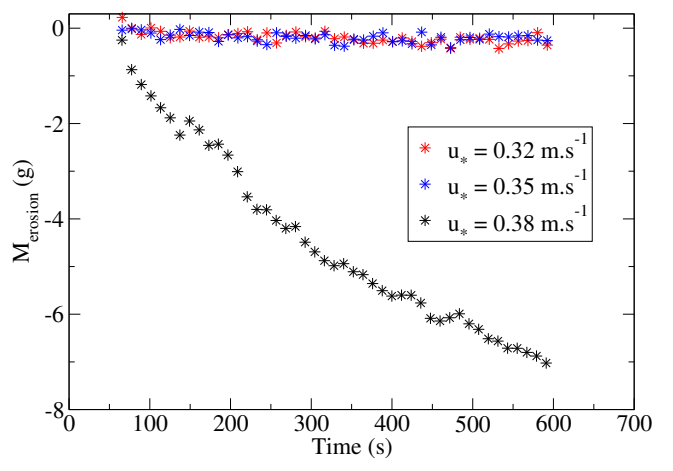

Figure 2. Temporal evolution of the mass of a dry sample in course of time for different friction speeds: For the two lowest friction speed, the erosion rate is constant during the experiment while for the highest friction speed the erosion rate is decreasing in course of time.

We generate 5 different mixture samples with gravimetric liquid content $w=0.025,0.05,0.075$ and $0.1 \%$. The mixing is made by successive dilution from a first sand sample filled with an important liquid content. The homogenization is easier than for sand-water mixture because of the low contact angle of the silicone oil with the sand. Due to cohesion, the filling of the box with the oil-sand mixture leads to weakly decreasing packing $\varphi$ although we employed the same procedure: $\varphi=0.565$ for $w=0 \%$ and $\varphi=0.550$ for $w=0.1 \%$. Test were also made to check to the oil-sand mixture is stable over several days under atmospheric condition: no mass change was measured.

For each sand sample, we measured the erosion rate $E=d M / S d t$ (where $M$ is the mass of the sand) in course of the experiment for a prescribed wind velocity. An experiment is typically run over $10 \mathrm{mn}$. This is repeated for different wind shear stresses close to the aerodynamic threshold. Typical temporal evolutions of the mass of a dry sand sample are shown in Fig. 2. We observe two different regimes. For friction speed close to the threshold, the erosion rate is constant over the whole duration of the experiment whereas for higher friction speed the erosion rate is decreasing over time. In the latter case, the total eroded mass during the experiment $\Delta M$ is rather significant $(\Delta M \approx 5 g)$ regarding the surface of the sand bed. This regime with a decreasing erosion rate is probably due to the finite size of the sand bed.

In the following, we restrain ourselves to wind shear stress where the erosion rate remains constant over time. This means that we concentrate on erosion regime close to the transport threshold.

\section{Experimental Results}

\subsection{Erosion rate near the aerodynamic transport threshold}

We first investigate the effect of the liquid content on the erosion rate to determine how the aerodynamic threshold varies with the liquid content. Fig. 3 presents the erosion rate as a function of the friction speed for various liquid content. We choose to plot the erosion rate in a semilogarithmic plot because of the wide range of magnitude of the erosion rate. This representation underlines that the erosion rate follows a nice exponential evolution of $\mathrm{E}$ with the friction speed. We define the transport threshold when the mass loss during the $10 \mathrm{mn}$ experiment is of the order 


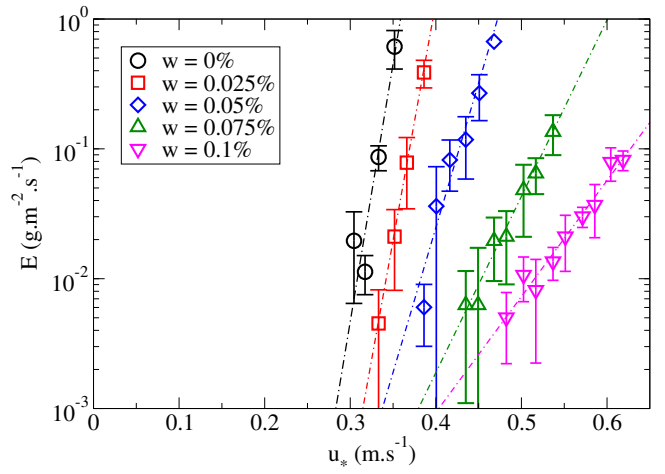

Figure 3. Erosion rate as a function of the friction speed $u_{*}$ for different liquid content $w$. The dash lines represent exponential fits to the data.

of the weight scale accuracy $\left(\Delta M<5 \cdot 10^{-2} g\right)$. Doing this, we obtain the values for the aerodynamic threshold as a function of the liquid content $w$ (see Fig. 4). The aerodynamic threshold exhibits a linear increase with $w$ :

$$
u_{* t w}=u_{* t d}(1+6.15 w) \text {. }
$$

This result is similar to that obtained by Hotta [6] with moist sand but differs significantly from that the logarithmic behavior found by Belly [5].

Important remarks follow. The threshold value obtained for dry sand is surprisingly high as compared with those from the literature for similar grain diameter: $u_{* t d}=$ $0.3 \mathrm{~m} \cdot \mathrm{s}^{-1}$ against $u_{* t d}=0.21 \mathrm{~m} \cdot \mathrm{s}^{-1}$ (see [3]). We believe that this high value comes from finite size effect. The sand bed is so short (i.e., $15 \mathrm{~cm}$ ) and this requires the transport to be triggered on short distances. It thus turns out that the threshold is probably dependent from the length of the sand bed. It can be interesting to express our results

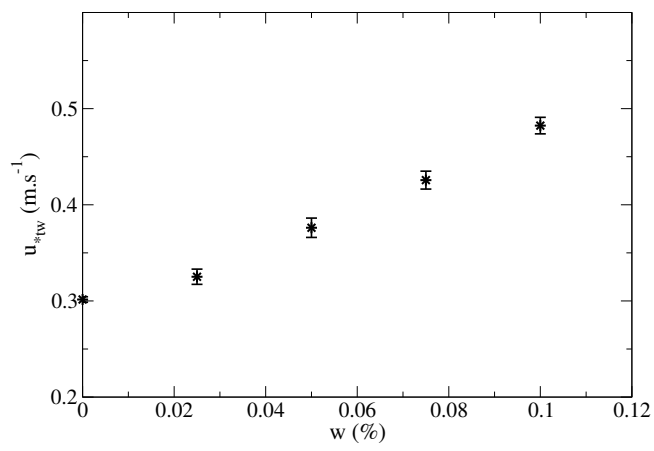

Figure 4. Aerodynamic threshold friction speed as a function of the water content

in terms of the cohesion force (or inverse Bond number) if one takes benefit Eq. 4. We plot in Fig. 5 the dimensionless cohesive force as a function of the liquid content $w$. As expected, we obtain an increase of the cohesion force with the liquid content. We can compare the values found with the one computed from the capillary force: $F_{c}=\pi D \gamma \cos \theta \approx 6.5 .10^{-6} N$ (where $\theta \approx 0$ is the contact angle) which corresponds to a dimensionless force $B_{o}^{-1} \approx 130$. The magnitude of the cohesion force found from erosion threshold is thus much smaller than that given by the capillary force. This probably mean that

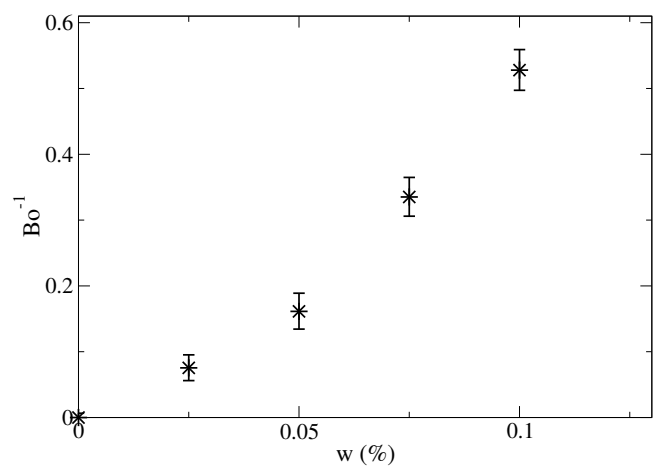

Figure 5. Dimensionless cohesive force $B_{o}^{-1}=F_{c} / F_{p}$ as a function of the liquid content $w$.

cohesion regime we investigate falls in the hygroscopic regime and not in the pendular one where capillary bridges are formed.

\subsection{Erosion by impact}

We also investigate erosion induced the impact of saltating particles. To do this, we installed a hopper filled with dry sand on the roof of the wind-tunnel close to the entrance. This allows us to prescribe a flow of saltating particle with a mass flux $Q_{i n}$. We set the mass flux value to a small value $Q_{\text {in }}=1.4 \mathrm{~g} . \mathrm{s}^{-1}$. With this incoming mass flux, the lowest friction speed we can investigate is $0.15 \mathrm{~m} / \mathrm{s}$. Below this value, a significant fraction of the incoming sand come to rest before the sample.

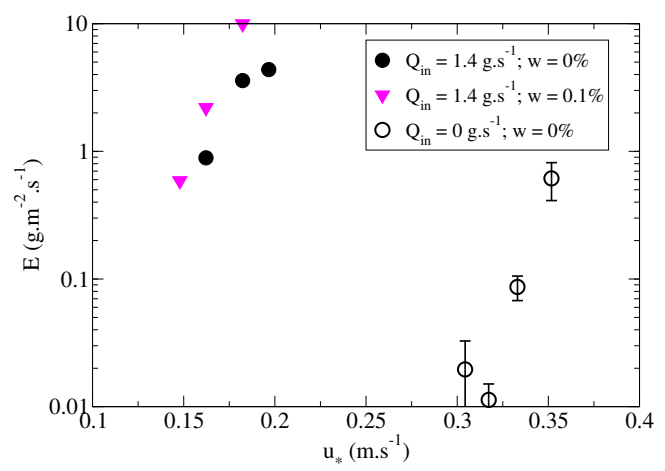

Figure 6. Impact erosion rate as a function of the friction speed for two different liquid content, $w=0$ and $0.1 \%$ respectively. The incoming mass flow rate is $Q_{i n}=1.4 \mathrm{~g} . \mathrm{s}^{-1}$. Open symbols correspond to data obtained for the aerodynamic erosion rate.

We measure the erosion rate as a function of the friction speed for different sand-oil mixtures subject to the same incoming flux $Q_{i n}$. Our preliminary results tend to show the impact threshold is not modified by cohesion in the range liquid content we investigated so far (see Fig. 6). 
This means that impact is very efficient to erode a cohesive bed in comparison with the aerodynamic entrainment. Recent numerical simulations on the impact process with a cohesive bed [9] indicate that the critical inverse Bond number above which cohesion alters the impact process is of the order of $B_{o}^{-1} \approx 5$. As shown in Fig. 5, the range of cohesion force is always below this critical value.

\section{Discussion and conclusion}

The modeling of aeolian transport of wet sand requires a good description of the erosion mechanisms in presence of cohesive capillary forces. The strength of these forces are dependent on the water content within the particle bed and it is therefore crucial to have a good control of the latter to get reliable data from wind-tunnel experiments. It is however a real challenge because as soon as a turbulent air flow runs over a wet sand bed, the water content decreases rapidly and non uniformly along and within the bed due to evaporation process. To circumvent this experimental difficulty, we used a non-volatile liquid to investigate the effects of cohesion on the aerodynamic or impact threshold. This ensures that the liquid content does not vary during an experiment. Our results first indicate that the aerodynamic threshold increases linearly with the liquid content as suggested by Hotta et al. [6]. Second, we do see any measurable effect of the cohesion on the impact threshold for the range of liquid content investigated so far. This last outcome thus indicates that erosion induced by impact is hardly affected by the bed cohesion. This finding may have strong implications in the context of aeolian transport of moist sand. We expect in particular that sand transport on wet sand, once initiated aerodynamically, can be sustained at very low wind strength and leads to substantial mass transport even below the aerodynamic threshold. This may explain the surprising high transport rates observed on sand beaches despite the large level of moisture [10].

This work has been supported by the French National Agency for Research (Grant No. ANR-17-CE01-0014).

\section{References}

[1] R.A.Bagnold, The physics of blown-sand and desert dunes. (Methuen, New York, 1941)

[2] W.S.Chepil, Soil Sci 60, 397-411 (1945)

[3] T.D.Ho, Etude expérimentale du transport de particules dans une couche limite turbulente. Thesis 4448 (Université de Rennes 1, Rennes, 2012)

[4] M.V.Carneiro, K.R.Rasmussen, H.J.Herrmann, Sci Rep-UK 5, 11109 (2015)

[5] P.Y.Belly, Sand movement by wind. Technical memorandum (United States Army Corps of Engineers, Coastal Engineering Research, Berkeley, California, 1962)

[6] S.Hotta, S.Kubota, S.Katori, K.Horikawa, Proceedings of the 19th Coastal Engineering Conference of the ASCE. 19, 1265-1281 (1984)

[7] C.McKenna-Neuman, W.G.Nickling, Can J Soil Sci 69, 79-96 (1989)

[8] Y.Kawata, Y.Tsuchiya, Proceedings of the Japan Society of Civil Engineers 249, 95-100 (1976)

[9] J.L.Ralaiarisoa, Influence de la cohésion sur le transport éolien de particules: Application au sable humide et à la neige (Université de Rennes 1, Rennes, 2021)

[10] R.G.D.Davidson-Arnott, K.MacQuarrie, T.Aagaard, Geomorphology 68, 115-129 (2005) 